\title{
DINÂMICA DE POPULAÇÕES DE ESPÉCIES ARBÓREAS EM UM FRAGMENTO DE FLORESTA OMBRÓFILA MISTA MONTANA EM LAGES, SANTA CATARINA
}

\author{
THE DYNAMICS OF TREE SPECIES POPULATION IN A MONTANE ARAUCARIA FOREST \\ FRAGMENT IN LAGES, SANTA CATARINA STATE
}

\author{
Bruna Salami $^{1}$ Pedro Higuchi ${ }^{2}$ Ana Carolina da Silva ${ }^{3}$ Tiago de Souza Ferreira ${ }^{4}$ \\ Amanda Koche Marcon ${ }^{1}$ Fernando Buzzi Júnior ${ }^{5}$ Marco Antônio Bento ${ }^{5}$
}

\begin{abstract}
RESUMO
O presente estudo teve como principal objetivo avaliar a dinâmica de populações de espécies arbóreas, em um fragmento de Floresta Ombrófila Mista Montana, no Município de Lages, Santa Catarina - SC, ao longo de um período de quatro anos (2008-2012). Para isto, para todas as espécies amostradas em 50 parcelas permanentes de $10 \times 20 \mathrm{~m}$, foram determinadas as guildas de regeneração [Pioneiras (P), Climácicas Exigentes em Luz (CEL) e Climácicas Tolerantes ao Sombreamento (CTS)] e as taxas de dinâmica (mortalidade, recrutamento, ganho e perda em área basal, mudança líquida em abundância e área basal). A existência de associação significativa entre as guildas de regeneração e os padrões de dinâmica foi verificada por meio de testes de qui-quadrado, aplicados a tabelas de contingência. Mudanças das participações relativas das guildas de regeneração entre os anos foram analisadas por meio de testes de proporção. No ano 2008 foram verificadas 87 espécies (P: 9, CEL: 64, CTS: 11 e não classificadas: 3), 1.841 ind.ha ${ }^{-1}$ e $36,17 \mathrm{~m}^{2} . \mathrm{ha}^{-1}$. Em 2012, verificaram-se 86 espécies (P: 9, CEL: 63, CTS: 11, não classificadas: 3), 1.882 ind.ha $^{-1}$ e 39,17m². $\mathrm{ha}^{-1}$. A maior parte das espécies apresentou ganho em abundância e área basal. Dentre as mais abundantes, as maiores taxas de recrutamento e ganho em área basal foram observadas para Banara tomentosa Clos, com, respectivamente, $5,43 \%$.ano ${ }^{-1}$ e $6,08 \%$.ano ${ }^{-1}$, e as maiores taxas de mortalidade e perda em área basal ocorreram para Zanthoxylum rhoifolium Lam., com, respectivamente, 7,75\%.ano ${ }^{-1}$ e 4,74\%.ano ${ }^{-1}$. Não foi verificada associação significativa entre os padrões de dinâmica observados e guildas de regeneração, cujas participações relativas na comunidade no período avaliado, permaneceram constantes. Desta forma, conclui-se que o fragmento florestal se encontra em fase de instabilidade estrutural, indicada pelo incremento no número de indivíduos e área basal, e estabilidade florística sucessional, em função da baixa substituição florística e manutenção da importância relativa das guildas de regeneração ao longo do tempo avaliado.

Palavras-chave: dinâmica florestal; sucessão ecológica; floresta com Araucária; guildas de regeneração.
\end{abstract}

\section{ABSTRACT}

The present study aimed to evaluate the tree species population dynamics in a fragment of Montane

1 Engenheira Florestal, MSc., Departamento de Engenharia Florestal, Universidade do Estado de Santa Catarina, Av. Luiz de Camões, 2090, CEP 88520-000, Lages (SC), Brasil. brunaflorestal@yahoo.com.br/ tiagoferreira@florestal.eng.br/ amandamarcon@yahoo.com.br

2 Engenheiro Florestal, Dr., Professor do Departamento de Engenharia Florestal, Universidade do Estado de Santa Catarina, Av. Luiz de Camões, 2090, CEP 88520-000, Lages (SC), Brasil. Bolsista do CNPq. higuchip@gmail.com

3 Engenheira Florestal, $\mathrm{Dr}^{\mathrm{a}}$., Professora do Departamento de Engenharia Florestal, Universidade do Estado de Santa Catarina, Av. Luiz de Camões, 2090, CEP 88520-000, Lages (SC), Brasil. Bolsista do CNPq. carol_sil4@yahoo.com.br

4 Engenheiro Florestal, MSc., Departamento de Engenharia Florestal, Universidade do Estado de Santa Catarina, Av. Luiz de Camões, 2090, CEP 88520-000, Lages (SC), Brasil. tiagoferreira@florestal.eng.br

5 Engenheiro Florestal, Departamento de Engenharia Florestal, Universidade do Estado de Santa Catarina, Av. Luiz de Camões, 2090, CEP 88520-000, Lages (SC), Brasil. buzzifjr@hotmail.com/ marco_a_bento@hotmail.com

Recebido para publicação em 18/07/2013 e aceito em 22/06/2015 
Araucaria Forest, in the municipality of Lages, Santa Catarina (SC) state, along a period of four years (2008-2012). For this, for all species sampled, in 50 permanent plots of $10 x 20 \mathrm{~m}$, the regeneration guilds [pioneer (P), light-demanding climax (LDC) and shade-tolerant climax (STC)] and dynamics rates were determined (mortality, recruitment, loss and gain in basal area, net change in abundance and basal area). The existence of significant association between regeneration guilds and dynamic rates was verified by chisquare tests, applied to contingency tables. Changes in relative participation of regeneration guilds between years were analyzed using proportion tests. In 2008, 87 species were found (P: 9, LDC: 64, STC: 11, nonclassified: 3), 1.841 ind.ha ${ }^{-1}$ and $36.17 \mathrm{~m}^{2}$.ha ${ }^{-1}$. In 2012, 86 species (P: 9, LDC: 63, STC: 11, non-classified: 3), 1.882 ind.ha $^{-1}$ and $39.17 \mathrm{~m}^{2}$.ha ${ }^{-1}$ were observed. Most species showed gains in basal area. Among the most abundant, the highest rates of recruitment and basal area gain were observed for Banara tomentosa Clos, with, respectively, 5.43\%.year ${ }^{-1}$ and $6.08 \%$.year $^{-1}$, and the highest rates of mortality and basal area loss occurred for Zanthoxylum rhoifolium Lam., with, respectively, $7.75 \%$.year ${ }^{-1}$ and $4.74 \%$.year ${ }^{-1}$. No significant association between the dynamics patterns and regeneration guilds was observed, whose relative participations in the community, during the evaluated period, were constants. Therefore, it is possible to conclude that the forest fragment is in the process of structural instability, indicated by the increment of the number of individuals and basal area, and floristic-successional stability, due to the low floristic substitution and maintenance of the relative importance of the regeneration guilds, along the evaluated period.

Keywords: forest dynamics; ecological succession; Araucaria Forest; regeneration guilds.

\section{INTRODUÇÃO}

A região fitoecológica da Floresta Ombrófila Mista (FOM) ou Floresta com Araucária caracterizase pela presença da Araucaria angustifolia (Bertol.) Kuntze em associações diversificadas, as quais compreendem agrupamentos de espécies com características próprias, formando estágios sucessionais distintos. A sua distribuição geográfica associa-se comumente com locais de elevada altitude e baixas temperaturas médias anuais, predominantemente na região do planalto meridional. Está dividida em quatro subformações considerando a influência de cursos d'água, e diferentes níveis de altitude: Aluvial, Submontana, Montana e Alto-Montana (IBGE, 2012).

Em função da intensa exploração madeireira que ocorreu no passado, da demanda por áreas agrícolas e pressões de origem antrópicas, como a criação de gado, a FOM encontra-se, assim como os demais remanescentes da Floresta Atlântica, fragmentada e com sua composição florística alterada (HIGUCHI et al., 2012; LINGNER et al., 2007). No entanto, mesmo estando atualmente isolados e perturbados, os remanescentes florestais desempenham funções ambientais de grande importância, como a proteção do solo e a manutenção dos cursos de água, a conexão entre fragmentos e reservas florestais e o sequestro de carbono atmosférico, mitigando os efeitos negativos das mudanças climáticas (HIGUCHI et al., 2012).

Considerando a importância da conservação dos remanescentes de FOM, é preciso conhecer a capacidade regenerativa e a produtividade dessas florestas alteradas por ações antrópicas, para que seja possível manejá-las de forma sustentável (SANQUETTA et al., 2003). Esta capacidade está intimamente relacionada com três processos demográficos fundamentais: o recrutamento, o crescimento e a mortalidade. Estes, por sua vez, são os alicerces do processo de dinâmica florestal. Os indivíduos que morrem são substituídos pelo crescimento dos indivíduos vizinhos ou pelo estabelecimento de novos indivíduos da mesma ou de diferentes espécies (MOSCOVICH, 2006). A percepção dessa mudança na composição estrutural e funcional das florestas, caracterizada pela dinâmica florestal, advém de estudos ao longo do tempo. Cada uma dessas mudanças interpreta-se como uma transição de um estágio sucessional para outro, que, de acordo com Machado (2005), podem ser verificadas tanto na demografia como na biomassa, tradicionalmente expressos como densidade e área basal.

Desta forma, considerando a importância dos estudos das mudanças ao longo do tempo que ocorrem em fragmentos florestais, o objetivo geral deste estudo foi avaliar a dinâmica de populações de espécies arbóreas em um fragmento de Floresta Ombrófila Mista Montana em Lages - SC, em um período de quatro anos, com o propósito de testar se (i) as guildas de regeneração apresentam diferentes padrões de dinâmica e (ii) a existência de mudança sucessional no fragmento estudado. 


\section{MATERIAL E MÉTODO}

\section{Caracterização da área de estudo}

O presente estudo foi realizado em um fragmento de Floresta Ombrófila Mista Montana, localizado no município de Lages - SC (2751'19,20”'S e 50¹0’33,39”W), conforme Figura 1. O fragmento possui uma área de aproximadamente 103,06 ha e altitude média de $940 \mathrm{~m}$ (altitude máxima =1.036 m e altitude mínima $=901 \mathrm{~m}$ ). De acordo com a estação meteorológica de Lages, administrada pela EPAGRI/CIRAM/INMET, a precipitação e a temperatura média anual (1970-2010) na região do fragmento é de, respectivamente, $1.682,80 \mathrm{~mm}$ e $15,9^{\circ} \mathrm{C}$ (HIGUCHI et al., 2012).
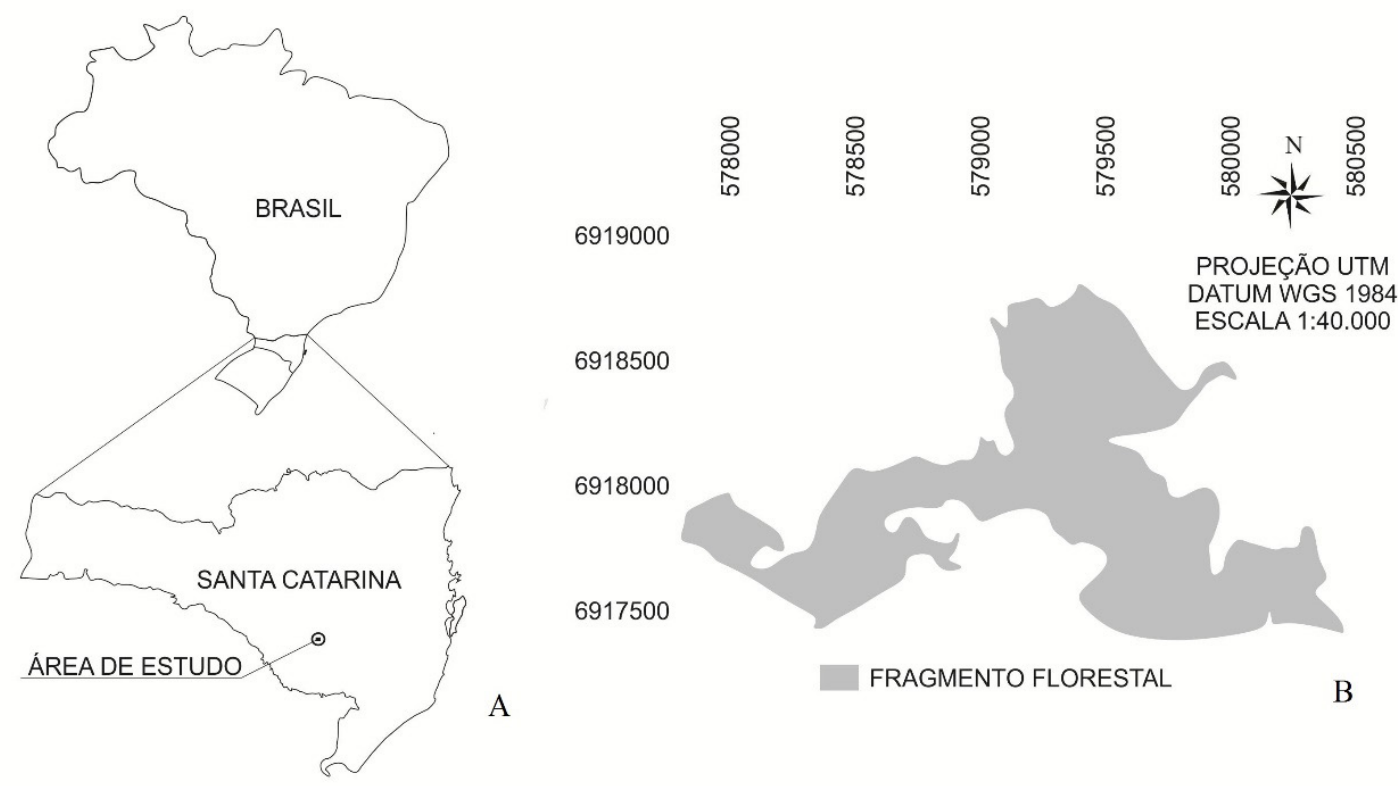

6918500

6917500

FIGURA 1: Localização geográfica (A) do fragmento de Floresta Ombrófila Mista Montana avaliado (B), em Lages, Santa Catarina.

FIGURE 1: Geographical location (A) of the studied Montane Araucaria Forest fragment (B) in Lages, Santa Catarina state.

A área de estudo encontra-se inserida em uma matriz composta por áreas agrícolas, silviculturais e de pastejo. O fragmento estudado possui um histórico de perturbação com corte seletivo e pastejo, porém, em 1986 foi protegida e se mantém em regeneração desde então.

\section{Amostragem da vegetação}

Esta área foi objeto de estudo florístico e estrutural realizado por Higuchi et al. (2012), contudo, com alterações no número de espécies, uma vez que a lista florística referente ao trabalho citado passou por revisão e correções. A comunidade arbórea, com diâmetro a altura do peito (DAP) maior ou igual a 5 $\mathrm{cm}$, foi amostrada no ano de 2008, em 50 parcelas permanentes de 10 × $20 \mathrm{~m}\left(200 \mathrm{~m}^{2}\right)$, totalizando 1 ha de área amostrada. As parcelas foram distribuídas de forma sistemática, distanciadas $30 \mathrm{~m}$ entre si dentro de cada estrato, definidos a partir da heterogeneidade ambiental da área. Em cada parcela foram registradas informações de todos os indivíduos vivos, coletando-se dados sobre a identidade botânica das espécies e do DAP, medido com fita métrica. Indivíduos com troncos múltiplos foram medidos quando a raiz da soma dos quadrados dos DAP foi maior do que $5 \mathrm{~cm}$. As identificações foram realizadas por meio de comparações em herbários, pareceres de especialistas e literatura especializada. As espécies foram classificadas nas famílias de acordo com o sistema APG III (ANGIOSPERM PHYLOGENY GROUP III, 2009).

No ano de 2012, após um intervalo de quatro anos, foi realizado o segundo inventário da comunidade 
arbórea, utilizando-se a mesma metodologia do inventário anterior. Assim, foram incorporados os novos indivíduos que atingiram a classe de diâmetro estipulada (DAP $\geq 5 \mathrm{~cm}$ ), sendo estes identificados, medidos e plaqueteados. Os indivíduos mortos foram registrados e os sobreviventes mensurados novamente.

\section{Análise dos dados}

Foram calculadas para a comunidade arbórea as taxas de mortalidade e recrutamento, as taxas de ganho e perda em área basal, com base no número de indivíduos e área basal, por meio dos modelos algébricos (LIEBERMAN et al., 1985; PRIMACK et al., 1985; SHEIL e MAY, 1996):

$$
\begin{gathered}
M=\left(1-\left(\left(N_{\mathrm{o}}-m\right) / N_{\mathrm{o}}\right)^{1 / t}\right) \times 100, \\
R=\left(1-\left(1-r / N_{t}\right)^{1 / t}\right) \times 100, \\
P=\left(1-\left[(A B-(A B \mathrm{~m}+A B \mathrm{~d})) / A B B_{\mathrm{o}}\right)^{1 / t}\right) \times 100 \\
G=\left(1-[1-(A B \mathrm{r}+A B \mathrm{~g}) / A B \mathrm{t})^{1 / t}\right) \times 100
\end{gathered}
$$

Em que: $\mathrm{M}=$ taxa de mortalidade anual; $\mathrm{R}=$ taxa de recrutamento anual; $\mathrm{P}=$ taxa de perda em área basal anual; $\mathrm{G}=$ taxa de ganho em área basal anual; $\mathrm{t}=$ intervalo de tempo entre inventários; $\mathrm{No}=$ número inicial de árvores; $\mathrm{Nt}=$ número final de árvores depois de $\mathrm{t} ; \mathrm{m}=$ número de árvores mortas; $\mathrm{r}=$ número de árvores recrutadas; $\mathrm{ABo}=$ área basal inicial; $\mathrm{ABt}=$ área basal final depois de $\mathrm{t} ; \mathrm{ABm}=$ área basal das árvores mortas; $\mathrm{ABd}=$ perda em área basal (redução diamétrica e perda parcial de troncos); $\mathrm{ABr}=$ área basal de árvores recrutadas; $\mathrm{ABg}=$ ganho em área basal (crescimento das árvores).

As taxas de mudança líquida para o número de indivíduos (Chn) e para a área basal (Chab) foram calculadas pelas equações, conforme Korning e Balslev (1994):

$$
\begin{gathered}
C h n=\left[\left(N_{\mathrm{t}} / N_{\mathrm{o}}\right)^{1 / t}-1\right] \times 100 \\
\text { Chab }=\left[\left(A B_{\mathrm{t}} / A B_{\mathrm{o}}\right)^{1 / t}-1\right] \times 100
\end{gathered}
$$

Com o propósito de auxiliar a identificação de padrões ecologicamente significativos, as espécies foram classificadas segundo suas guildas de regeneração: Pioneira, Clímax Exigente em Luz e Clímax Tolerante à Sombra. As classificações das espécies basearam-se nos trabalhos de Lindenmaier e Budke (2006) e Leyser et al. (2012) e a partir de observações no campo. Para verificar a existência de associação significativa entre os padrões de dinâmica das populações (incremento, estabilidade ou decremento em abundância em área basal) e as guildas de regeneração, foram realizados testes de qui-quadrado, aplicados a tabelas de contingência. Mudanças nas participações relativas das guildas ao longo do tempo foram verificadas por meio de testes de proporção. Todas as análises foram realizadas no programa estatístico $\mathrm{R}$ (R DEVELOPMENT CORE TEAM, 2012).

\section{RESULTADOS E DISCUSSÃO}

Os resultados demonstram que a comunidade se encontra em processo de estruturação, com incremento em abundância e em área basal. No período de 2008 a 2012, as taxas de mortalidade e de recrutamento foram respectivamente de $1,23 \%$.ano ${ }^{-1}$ e $1,77 \%$.ano ${ }^{-1}$, o que resultou num aumento de 1.841 a 1.882 ind.ha $^{-1}$, representando uma mudança líquida positiva de $2,18 \%$. A taxa de ganho em área basal, de $2,93 \%$ ano $^{-1}$, foi aproximadamente três vezes superior à taxa de perda, de $0,97 \%$ ano $^{-1}$, resultando em um incremento de $36,17 \mathrm{~m}^{2}$.ha-1 para $39,17 \mathrm{~m}^{2}$.ha-1, que representou uma mudança líquida positiva de 6,67\%. Em relação à riqueza, foi observada a redução de apenas uma espécie, de 87 em 2008 para 86 em 2012, o que representou o saldo do ganho de uma espécie (Myrsine parvula (Mez) Otegui) e da perda de duas (Machaerium stipitatum Vogel e Myrceugenia oxysepala (Burret) D.Legrand \& Kausel). Do total de espécies encontradas no período, 65 foram classificadas como Clímax Exigente em Luz, 11 como Clímax Tolerante à Sombra e nove classificadas como Pioneiras (Tabela 1).

Pode-se interpretar o resultado encontrado da dinâmica da comunidade arbórea sob o contexto silvigenético (HALLÉ et al., 2005). O aumento em abundância e área basal sugere que a floresta se encontra 
TABELA 1: Número de indivíduos e área basal das populações de espécies arbóreas amostradas em um fragmento de Floresta Ombrófila Mista Montana, no Município de Lages - SC, ordenadas em função da abundância em 2008, para os anos de 2008 e 2012.

TABLE 1: Number of individuals and basal area of tree species populations sampled in a Montane Araucaria Forest, in the municipality of Lages, SC state, sorted by abundance in 2008, for the period of 2008 and 2012.

\begin{tabular}{|c|c|c|c|c|c|c|c|}
\hline Espécies & $\begin{array}{c}\mathrm{N} 1 \\
\text { (ind.ha }^{-1} \text { ) }\end{array}$ & $\begin{array}{c}\mathrm{N} 2 \\
\text { (ind.ha }^{-1} \text { ) }\end{array}$ & MLN (\%) & $\operatorname{AB} 1\left(m^{2} \cdot h^{-1}\right)$ & $\operatorname{AB} 2\left(\mathrm{~m}^{2} \cdot \mathrm{ha}^{-1}\right)$ & MLAB (\%) & Guildas \\
\hline Jacaranda puberula Cham. & 172 & 157 & $-2,26$ & 1,5427 & 1,4495 & $-1,55$ & CEL \\
\hline Casearia decandra Jacq. & 147 & 166 & 3,09 & 0,8895 & 0,9477 & 1,60 & CTS \\
\hline $\begin{array}{l}\text { Araucaria angustifolia (Bertol.) } \\
\text { Kuntze }\end{array}$ & 125 & 128 & 0,59 & 5,2692 & 6,1535 & 3,95 & CEL \\
\hline Lithrea brasiliensis Marchand & 100 & 97 & $-0,76$ & 3,6391 & 3,8855 & 1,65 & CEL \\
\hline Matayba elaeagnoides Radlk. & 84 & 88 & 1,17 & 2,2035 & 2,3907 & 2,06 & CEL \\
\hline Myrsine umbellata Mart. & 70 & 74 & 1,40 & 0,7040 & 0,8086 & 3,52 & CEL \\
\hline $\begin{array}{l}\text { Podocarpus lambertii Klotzsch ex } \\
\text { Endl. }\end{array}$ & 69 & 71 & 0,72 & 1,4781 & 1,4858 & 0,13 & CEL \\
\hline Sapium glandulosum (L.) Morong & 66 & 63 & $-1,16$ & 1,2575 & 1,3563 & 1,91 & CEL \\
\hline Casearia obliqua Spreng. & 55 & 52 & $-1,39$ & 0,9153 & 0,9077 & $-0,21$ & CEL \\
\hline Cupania vernalis Cambess. & 53 & 58 & 2,28 & 0,6198 & 0,6876 & 2,63 & CEL \\
\hline Duranta vestita Cham. & 50 & 52 & 0,99 & 0,3175 & 0,3372 & 1,51 & $\mathrm{P}$ \\
\hline Dicksonia sellowiana Hook. & 38 & 42 & 2,53 & 1,5347 & 1,6830 & 2,33 & CTS \\
\hline Lamanonia ternata Vell. & 37 & 38 & 0,67 & 1,6689 & 1,8238 & 2,24 & CEL \\
\hline Ocotea pulchella (Nees \& Mart.) Nees & 34 & 33 & $-0,74$ & 1,6026 & 1,6764 & 1,13 & CEL \\
\hline $\begin{array}{l}\text { Allophylus guaraniticus (A.St.-Hil.) } \\
\text { Radlk. }\end{array}$ & 32 & 33 & 0,77 & 0,2200 & 0,2426 & 2,48 & CEL \\
\hline Calyptranthes concinna DC. & 30 & 32 & 1,63 & 0,2205 & 0,2185 & $-0,22$ & CTS \\
\hline Zanthoxylum rhoifolium Lam. & 29 & 23 & $-5,63$ & 0,3549 & 0,3260 & $-2,09$ & CEL \\
\hline $\begin{array}{l}\text { Zanthoxylum kleinii (R.S.Cowan) } \\
\text { P.G.Waterman }\end{array}$ & 27 & 25 & $-1,91$ & 0,4464 & 0,4877 & 2,24 & CEL \\
\hline $\begin{array}{l}\text { Dasyphyllum brasiliense (Spreng.) } \\
\text { Cabrera }\end{array}$ & 26 & 25 & $-0,98$ & 1,2699 & 1,3420 & 1,39 & CEL \\
\hline Banara tomentosa Clos & 25 & 30 & 4,66 & 0,1162 & 0,1451 & 5,72 & CEL \\
\hline Eugenia pluriflora DC. & 23 & 26 & 3,11 & 0,2019 & 0,2183 & 1,96 & CEL \\
\hline Myrcia guianensis (Aubl.) DC. & 23 & 24 & 1,07 & 0,2007 & 0,1979 & $-0,36$ & CEL \\
\hline Drimys brasiliensis Miers & 22 & 22 & 0 & 0,1184 & 0,1264 & 1,64 & CEL \\
\hline Prunus myrtifolia (L.) Urb. & 22 & 22 & 0 & 0,6778 & 0,7674 & 3,15 & CEL \\
\hline Solanum sanctae-catharinae Dunal & 22 & 21 & $-1,16$ & 0,3722 & 0,4123 & 2,59 & $\mathrm{P}$ \\
\hline Xylosma ciliatifolia (Clos) Eichler & 22 & 24 & 2,20 & 0,1606 & 0,1855 & 3,66 & CEL \\
\hline Ilex theezans Mart. ex Reissek & 21 & 20 & $-1,21$ & 0,3814 & 0,3735 & $-0,53$ & CEL \\
\hline $\begin{array}{l}\text { Vernonanthura discolor (Spreng.) } \\
\text { H.Rob. }\end{array}$ & 21 & 22 & 1,17 & 1,0205 & 1,1780 & 3,65 & $\mathrm{P}$ \\
\hline Schinus terebinthifolius Raddi & 20 & 19 & $-1,27$ & 0,1525 & 0,1478 & $-0,77$ & CEL \\
\hline $\begin{array}{l}\text { Blepharocalyx salicifolius (Kunth) } \\
\text { O.Berg }\end{array}$ & 19 & 20 & 1,29 & 0,1701 & 0,1940 & 3,34 & CEL \\
\hline Machaerium paraguariense Hassl. & 19 & 19 & 0 & 0,3513 & 0,3208 & $-2,25$ & CEL \\
\hline Myrcia hatschbachii D.Legrand & 18 & 18 & 0 & 0,2758 & 0,3022 & 2,31 & CEL \\
\hline Annona rugulosa (Schltdl.) H.Rainer & 17 & 21 & 5,42 & 0,1128 & 0,1383 & 5,23 & CEL \\
\hline
\end{tabular}


TABELA 1: Continuação...

TABLE 1: Continued...

\begin{tabular}{|c|c|c|c|c|c|c|c|}
\hline Espécies & $\begin{array}{c}\mathrm{N} 1 \\
\left.\text { (ind.ha- }{ }^{-1}\right)\end{array}$ & $\begin{array}{c}\mathrm{N} 2 \\
\text { (ind.ha- }\end{array}$ & MLN (\%) & $\operatorname{AB} 1\left(\mathrm{~m}^{2} \cdot \mathrm{ha}^{-1}\right)$ & $\operatorname{AB} 2\left(\mathrm{~m}^{2} \cdot \mathrm{ha}^{-1}\right)$ & MLAB (\%) & Guildas \\
\hline Gymnanthes klotzschiana Müll.Arg. & 17 & 16 & $-1,50$ & 0,2716 & 0,2725 & 0,09 & CEL \\
\hline Myrcia palustris DC. & 16 & 19 & 4,39 & 0,1237 & 0,1333 & 1,90 & CEL \\
\hline $\begin{array}{l}\text { Myrsine coriacea (Sw.) R.Br. ex } \\
\text { Roem. \& Schult. }\end{array}$ & 16 & 17 & 1,53 & 0,3448 & 0,3769 & 2,25 & CEL \\
\hline Inga sp. & 14 & 14 & 0 & 0,1889 & 0,1987 & 1,27 & - \\
\hline Roupala montana Aubl. & 14 & 15 & 1,74 & 0,2249 & 0,2498 & 2,66 & CTS \\
\hline $\begin{array}{l}\text { Celtis iguanaea } \\
\text { (Jacq.) Sarg. }\end{array}$ & 12 & 9 & $-6,94$ & 0,1117 & 0,0999 & $-2,75$ & $\mathrm{P}$ \\
\hline $\begin{array}{l}\text { Cinnamoтит атоепит (Nees \& } \\
\text { Mart.) Kosterm. }\end{array}$ & 12 & 12 & 0 & 0,6779 & 0,7637 & 3,03 & CEL \\
\hline Symplocos uniflora (Pohl) Benth. & 12 & 12 & 0 & 0,1524 & 0,1629 & 1,69 & CEL \\
\hline Dalbergia frutescens (Vell.) Britton & 11 & 14 & 6,21 & 0,1127 & 0,1407 & 5,71 & CEL \\
\hline $\begin{array}{l}\text { Moquiniastrum polymorphum (Less.) } \\
\text { G. Sancho }\end{array}$ & 11 & 11 & 0 & 0,3760 & 0,3965 & 1,34 & $P$ \\
\hline Oreopanax fulvus Marchal & 10 & 11 & 2,41 & 0,0989 & 0,1107 & 2,86 & CEL \\
\hline Cedrela fissilis Vell. & 9 & 9 & 0 & 0,1491 & 0,1711 & 3,51 & CEL \\
\hline Sebastiania brasiliensis Spreng. & 9 & 10 & 2,67 & 0,0570 & 0,0732 & 6,45 & CEL \\
\hline Styrax leprosus Hook. \& Arn. & 9 & 9 & 0 & 0,1786 & 0,2318 & 6,74 & CEL \\
\hline $\begin{array}{l}\text { Campomanesia xanthocarpa (Mart.) } \\
\text { O.Berg }\end{array}$ & 8 & 10 & 5,74 & 0,2143 & 0,2299 & 1,78 & CTS \\
\hline $\begin{array}{l}\text { Dasyphyllum spinescens (Less.) } \\
\text { Cabrera }\end{array}$ & 8 & 9 & 2,99 & 0,5150 & 0,5613 & 2,17 & CEL \\
\hline Scutia buxifolia Reissek & 8 & 9 & 2,99 & 0,1353 & 0,1601 & 4,30 & CEL \\
\hline $\begin{array}{l}\text { Allophylus edulis (A.St.-Hil., et al.) } \\
\text { Hieron. ex Niederl. }\end{array}$ & 7 & 11 & 11,96 & 0,0466 & 0,0662 & 9,17 & CEL \\
\hline Ilex dumosa Reissek & 7 & 7 & 0 & 0,1016 & 0,1112 & 2,30 & CTS \\
\hline Myrcia laruotteana Cambess. & 7 & 8 & 3,39 & 0,0625 & 0,0730 & 3,94 & CEL \\
\hline $\begin{array}{l}\text { Nectandra megapotamica (Spreng.) } \\
\text { Mez }\end{array}$ & 7 & 8 & 3,39 & 0,1516 & 0,1847 & 5,05 & CEL \\
\hline Clethra scabra Pers. & 6 & 6 & 0 & 0,1082 & 0,1175 & 2,08 & $\mathrm{P}$ \\
\hline Escallonia bifida Link \& Otto & 6 & 5 & $-4,46$ & 0,0841 & 0,0850 & 0,27 & $\mathrm{P}$ \\
\hline Coutarea hexandra (Jacq.) K.Schum. & 5 & 5 & 0 & 0,0658 & 0,0826 & 5,86 & CEL \\
\hline Erythroxylum deciduum A.St.-Hil. & 5 & 5 & 0 & 0,0981 & 0,1069 & 2,16 & CEL \\
\hline Eugenia pyriformis Cambess. & 5 & 5 & 0 & 0,0626 & 0,0633 & 0,27 & CEL \\
\hline Ilex brevicuspis Reissek & 5 & 5 & 0 & 0,1096 & 0,1160 & 1,44 & CEL \\
\hline Maytenus dasyclada Mart. & 4 & 5 & 5,74 & 0,0590 & 0,0580 & $-0,40$ & CEL \\
\hline Xylosma tweediana (Clos) Eichler & 4 & 4 & 0 & 0,0126 & 0,0144 & 3,30 & CEL \\
\hline Myrrhinium atropurpureum Schott & 3 & 3 & 0 & 0,0344 & 0,0322 & $-1,65$ & CEL \\
\hline NI & 3 & 4 & 7,46 & 0,0393 & 0,0341 & $-3,46$ & - \\
\hline Solanum pabstii L.B.Sm. \& Downs & 3 & 1 & 24,02 & 0,0163 & 0,0038 & 30,32 & CEL \\
\hline Eugenia uniflora $\mathrm{L}$. & 2 & 2 & 0 & 0,0286 & 0,0327 & 3,46 & CEL \\
\hline Ilex microdonta Reissek & 2 & 2 & 0 & 0,0334 & 0,0354 & 1,46 & CTS \\
\hline Nectandra lanceolata Nees & 2 & 2 & 0 & 0,0599 & 0,0718 & 4,65 & CEL \\
\hline Acca sellowiana (O.Berg) Burret & 1 & 1 & 0 & 0,0033 & 0,0033 & 0,69 & $\mathrm{P}$ \\
\hline
\end{tabular}

Continua... 
TABELA 1: Continuação...

TABLE 1: Continued...

\begin{tabular}{|c|c|c|c|c|c|c|c|}
\hline Espécies & $\begin{array}{c}\mathrm{N} 1 \\
\text { (ind.ha } \\
\end{array}$ & $\begin{array}{c}\mathrm{N} 2 \\
\text { (ind.ha- }\end{array}$ & MLN (\%) & $\operatorname{AB} 1\left(\mathrm{~m}^{2} \cdot \mathrm{ha}^{-1}\right)$ & $\operatorname{AB} 2\left(\mathrm{~m}^{2} \cdot \mathrm{ha}^{-1}\right)$ & MLAB (\%) & Guildas \\
\hline Asteraceae 1 & 1 & 1 & 0 & 0,0223 & 0,0215 & $-0,95$ & - \\
\hline $\begin{array}{l}\text { Citronella paniculata (Mart.) } \\
\text { R.A.Howard }\end{array}$ & 1 & 1 & 0 & 0,0038 & 0,0038 & 0 & CEL \\
\hline Eugenia uruguayensis Cambess. & 1 & 1 & 0 & 0,0022 & 0,0022 & 0 & CEL \\
\hline Handroanthus albus (Cham.) Mattos & 1 & 1 & 0 & 0,0323 & 0,0367 & 3,24 & CEL \\
\hline Ilex paraguariensis A.St.-Hil. & 1 & 1 & 0 & 0,0038 & 0,0048 & 6,20 & CTS \\
\hline Lauraceae 1 & 1 & 1 & 0 & 0,0272 & 0,0290 & 1,61 & - \\
\hline Machaerium stipitatum Vogel & 1 & 0 & -100 & 0,0314 & 0 & -100 & CEL \\
\hline Maytenus boaria Molina & 1 & 1 & 0 & 0,0121 & 0,0121 & 0 & CEL \\
\hline Mimosa scabrella Benth. & 1 & 1 & 0 & 0,0625 & 0,0673 & 1,90 & $P$ \\
\hline $\begin{array}{l}\text { Myrceugenia euosma (O.Berg) } \\
\text { D.Legrand }\end{array}$ & 1 & 1 & 0 & 0,0032 & 0,0037 & 3,68 & CEL \\
\hline $\begin{array}{l}\text { Myrcengenia myrcioides (Cambess.) } \\
\text { O.Berg }\end{array}$ & 1 & 1 & 0 & 0,0056 & 0,0064 & 3,64 & CEL \\
\hline $\begin{array}{l}\text { Myrceugenia oxysepala (Burret) } \\
\text { D.Legrand \& Kausel }\end{array}$ & 1 & 0 & -100 & 0,0027 & 0 & -100 & CEL \\
\hline Myrcia multiflora (Lam.) DC. & 1 & 1 & 0 & 0,0756 & 0,0877 & 3,77 & CTS \\
\hline $\begin{array}{l}\text { Myrcianthes gigantea (D.Legrand) } \\
\text { D.Legrand }\end{array}$ & 1 & 1 & 0 & 0,0020 & 0,0022 & 2,79 & CEL \\
\hline Myrciaria delicatula (DC.) O.Berg & 1 & 1 & 0 & 0,0029 & 0,0028 & $-1,23$ & CTS \\
\hline Myrtaceae sp. & 1 & 1 & 0 & 0,0074 & 0,0074 & $-0,07$ & - \\
\hline Ocotea diospyrifolia (Meisn.) Mez & 1 & 1 & 0 & 0,0276 & 0,0390 & 9,02 & CTS \\
\hline $\begin{array}{l}\text { Quillaja brasiliensis (A.St.-Hil. \& } \\
\text { Tul.) Mart. }\end{array}$ & 1 & 1 & 0 & 0,0048 & 0,0055 & 3,41 & CEL \\
\hline Rhamnus sphaerosperma $\mathrm{Sw}$. & 1 & 1 & 0 & 0,0022 & 0,0023 & 0,89 & CEL \\
\hline Myrsine parvula (Mez) Otegui & 0 & 1 & 100 & 0 & 0,0031 & 100 & CEL \\
\hline Total & 1841 & 1882 & & 36,1651 & 39,1708 & & \\
\hline
\end{tabular}

Em que: N1 = Número de Indivíduos no ano de 2008; N2 = Número de Indivíduos no ano de 2012; ML = Mudança Líquida; AB1 = Área Basal no ano de 2008; AB2 = Área Basal no ano de 2012; CEL = Clímax Exigente em Luz; CTS $=$ Clímax Tolerante à Sombra; $\mathrm{P}=$ Pioneira.

em processo de "construção", de forma que o clímax estrutural ainda não tenha sido alcançado. Nestas circunstâncias, observa-se a estruturação da floresta, indicando, segundo Sanquetta (2002) e Mognon (2011), que a mesma, ao demandar o carbono atmosférico para produção de biomassa, funcione como sumidouro de dióxido de carbono $\left(\mathrm{CO}_{2}\right)$.

O padrão observado de estruturação do componente arbóreo parece ser um padrão recorrente em estudos de dinâmica realizados em fragmentos de Floresta Ombrófila Mista (FOM) (DURIGAN, 1999; SANQUETTA et al., 2003; FORMENTO et al., 2004). Figueiredo-Filho et al. (2010), analisando a dinâmica de 11 áreas de FOM, distribuídas nos três estados do Sul do Brasil, verificaram que, de modo geral, ocorreu incremento em área basal, com a taxa de mortalidade variando entre 1 a $2 \%$, e a taxa de ingresso com uma média de quase $3 \%$ ao ano. Variações das taxas de dinâmica entre diferentes áreas, em escala regional, refletem diferenças nos estágios sucessionais, variações ambientais e, como sugerido pelos autores, diferenças no histórico de perturbação. Desta forma, a partir do resultado encontrado no presente estudo e da literatura existente, pode-se inferir que os remanescentes de FOM do Sul do Brasil, após um período intenso de exploração predatória durante o ciclo da madeira, encontram-se atualmente em fase de recuperação estrutural. 
Ainda, de acordo com a Tabela 1, dentre as 20 espécies mais abundantes, as que apresentaram maior incremento em número de indivíduos foram Casearia decandra (3,09\%), Myrsine umbellata (1,40\%), Matayba elaeagnoides (1,17\%), Podocarpus lambertii (0,72\%) e Araucaria angustifolia $(0,59 \%)$, todas classificadas como Clímax Exigente de Luz, exceto $C$. decandra, que é Clímax Tolerante à Sombra. Em relação à área basal, as que apresentaram maior ganho foram a Araucaria angustifolia (3,95\%), Myrsine umbellata (3,52\%), Matayba elaeagnoides (2,06\%), Lithrea brasiliensis (1,65\%) e Casearia decandra $(1,60 \%)$. Desta forma, as espécies supracitadas foram as que mais contribuíram para a estruturação florestal, tanto em aumento de número de indivíduos quanto incremento de área basal. Araucaria angustifolia, também apresentou ganho em área basal destacado em outras áreas de FOM no Sul do Brasil (FIGUEIREDO-FILHO et al., 2010). Formento, Schorn e Ramos (2004), na mesma bacia hidrográfica do presente estudo, além de Araucaria angustifolia, também observaram aumento da importância relativa de Lithrea brasiliensis, Matayba elaeagnoides e Ocotea pulchella. Desta forma, fica evidente a elevada capacidade de crescimento destas espécies e o potencial de seu manejo visando à exploração sustentável.

As espécies que se destacaram em relação às mudanças líquidas negativas em número de indivíduos foram Jacaranda puberula (-2,26\%), Casearia obliqua (-1,39\%), Sapium glandulosum (-1,16\%), Lithrea brasiliensis $(-0,76 \%)$ e Ocotea pulchella $(-0,74 \%)$, todas classificadas como CEL. As que se destacaram negativamente em relação à mudança líquida quanto à área basal foram Zanthoxylum rhoifolium $(-2,09 \%)$,

TABELA 2: Taxas de mortalidade e recrutamento, e taxas de ganho e perda em área basal, com base no número de indivíduos e área basal das 20 espécies mais abundantes em um fragmento de FOM, em Lages - SC.

TABLE 2: Mortality and recruitment rates, and basal area gain and loss rates, based on the number of individuals and basal area of the 20 most abundant species in a Araucaria Forest fragment, in the municipality of Lages, SC state.

\begin{tabular}{|c|c|c|c|c|}
\hline Espécies & $\begin{array}{c}\text { TxMort } \\
\left(\% \cdot \text { ano }^{-1}\right)\end{array}$ & $\begin{array}{c}\text { TxRecr } \\
\left(\% . \text {.no }^{-1}\right)\end{array}$ & $\begin{array}{l}\text { TxGanho } \\
\left(\% . \text { ano }^{-1}\right) \\
\end{array}$ & $\begin{array}{r}\text { TxPerda } \\
\left(\% \text {.ano }{ }^{-1}\right) \\
\end{array}$ \\
\hline Jacaranda puberula Cham. & 4,01440 & 1,79959 & 3,05737 & 4,55575 \\
\hline Casearia decandra Jacq. & 0,86141 & 3,82879 & 4,50728 & 2,98195 \\
\hline Araucaria angustifolia (Bertol.) Kuntze & 0,20060 & 0,79058 & 4,02722 & 0,23186 \\
\hline Lithrea brasiliensis Marchand & 1,01536 & 0,25873 & 2,24984 & 0,63583 \\
\hline Matayba elaeagnoides Radlk. & 0,90507 & 2,05087 & 3,08231 & 1,08680 \\
\hline Myrsine umbellata Mart. & 1,08909 & 2,45371 & 4,31025 & 0,93838 \\
\hline Podocarpus lambertii Klotzsch ex Endl. & 0,36430 & 1,07350 & 0,95319 & 0,82510 \\
\hline Sapium glandulosum (L.) Morong & 1,15626 & 0 & 2,25043 & 0,38372 \\
\hline Casearia obliqua Spreng. & 1,39245 & 0 & 1,83649 & 2,04180 \\
\hline Cupania vernalis Cambess. & 0 & 2,22857 & 3,04783 & 0,49793 \\
\hline Duranta vestita Cham. & 1,53498 & 2,49573 & 3,98875 & 2,53852 \\
\hline Dicksonia sellowiana Hook. & 0 & 2,47104 & 3,12214 & 0,86227 \\
\hline Lamanonia ternata Vell. & 0 & 0,66449 & 2,37988 & 0,19075 \\
\hline Ocotea pulchella (Nees \& Mart.) Nees. & 1,50419 & 0,76634 & 1,89175 & 0,78070 \\
\hline Allophylus guaraniticus (A.St.-Hil.) Radlk. & 0,79058 & 1,55086 & 4,89983 & 2,53939 \\
\hline Calyptranthes concinna DC. & 0,84396 & 2,43097 & 1,96296 & 2,18189 \\
\hline Zanthoxylum rhoifolium Lam. & 7,75235 & 2,24863 & 2,70215 & 4,74018 \\
\hline Zanthoxylum kleinii (R.S.Cowan) P.G.Waterman & 1,90563 & 0 & 3,23251 & 1,06854 \\
\hline Dasyphyllum brasiliense (Spreng.) Cabrera & 0,97573 & 0 & 1,96512 & 0,60136 \\
\hline Banara tomentosa Clos & 1,01536 & 5,42584 & 6,07951 & 0,70650 \\
\hline
\end{tabular}

Em que: TxMort $=$ Taxa de mortalidade; TxRecr $=$ Taxa de recrutamento; TxGanho $=$ Taxa de ganho em área basal; TxPerda $=$ Taxa de perda em área basal. 
Jacaranda puberula (-1,55\%), Myrcia guianensis (-0,36\%), Calyptranthes concinna (-0,22\%) e Casearia obliqua (-0,21\%) classificadas como CEL, exceto Calyptranthes concinna, que é classificado como CTS.

Dentre as mais abundantes, Banara tomentosa $\left(5,43 \%\right.$.ano $\left.{ }^{-1}\right)$, Casearia decandra $\left(3,83 \%\right.$.ano $\left.{ }^{-1}\right)$, Duranta vestita $\left(2,50 \% \cdot\right.$ ano $\left.^{-1}\right)$, Dicksonia sellowiana $\left(2,47 \%\right.$.ano $\left.{ }^{-1}\right)$ e Myrsine umbellata $\left(2,45 \%\right.$. ano $\left.^{-1}\right)$ possuem os maiores valores de taxa de recrutamento da área (Tabela 2).

As espécies Zanthoxylum rhoifolium $\left(7,75 \%\right.$.ano $\left.{ }^{-1}\right)$, Jacaranda puberula $\left(4,01 \%\right.$.ano $\left.{ }^{-1}\right)$, Zanthoxylum kleinii $\left(1,91 \%\right.$.ano $\left.{ }^{-1}\right)$, Duranta vestita $\left(1,53 \%\right.$. ano $\left.^{-1}\right)$ e Ocotea pulchella $\left(1,50 \%\right.$.ano $\left.{ }^{-1}\right)$ apresentaram as maiores taxas de mortalidade encontradas na área. As espécies que mais se destacaram quanto ao ganho em área basal foram Banara tomentosa $\left(6,08 \%\right.$. ano $\left.{ }^{-1}\right)$, Allophylus guaraniticus $\left(4,90 \%\right.$.ano $\left.{ }^{-1}\right)$, Casearia decandra $\left(4,51 \%\right.$.ano $\left.{ }^{-1}\right)$, Myrsine umbellata $\left(4,31 \%\right.$.ano $\left.{ }^{-1}\right)$ e Araucaria angustifolia $\left(4,03 \%\right.$.ano $\left.{ }^{-1}\right)$. As espécies que mais se destacaram quanto à perda em área basal foram Zanthoxylum rhoifolium $(4,74 \%$. ano $\left.^{-1}\right)$, Jacaranda puberula $\left(4,56 \%\right.$.ano $\left.{ }^{-1}\right)$, Casearia decandra $\left(2,98 \%\right.$.ano $\left.{ }^{-1}\right)$, Allophylus guaraniticus $\left(2,54 \%\right.$.ano $\left.{ }^{-1}\right)$ e Duranta vestita $\left(2,54 \%\right.$.ano $\left.{ }^{-1}\right)$. Desta maneira, é possível observar que os valores de taxas de recrutamento, mortalidade e ganho e perda em área basal variam entre as espécies mais abundantes, indicando o comportamento ecológico diferenciado (SERRÃO et al., 2003; ROIK et al., 2012).

Em relação à abundância, a maioria das espécies, 36, não apresentou mudança líquida, mantendo o tamanho populacional entre os anos avaliados, 32 espécies apresentaram mudança líquida positiva e 17 apresentaram mudança negativa (Tabela 3).

Das 17 espécies que apresentaram declínio do número de indivíduos, três foram classificadas como Pioneiras e 14 como Clímax Exigentes em Luz. Das 32 espécies com aumento do número de indivíduos, duas eram Pioneiras, 25 foram classificadas como Clímax Exigentes em Luz e cinco como Clímax Tolerante à Sombra. Das que se apresentaram estáveis, quatro eram Pioneiras, 26 Clímax Exigentes em Luz e seis classificadas como Clímax Tolerante à Sombra. O teste de qui-quadrado demonstrou que não existiu associação significativa entre os padrões de dinâmica e a classificação das espécies em grupos quanto às guildas de regeneração $\left(\chi^{2}=4,3156, p=0,365\right)$. Isto demonstra que a alteração do número de indivíduos observada não apresentou associação significativa com as guildas de regeneração.

Em relação à área basal, o maior número de espécies, 67, apresentou mudança líquida positiva, 15 apresentaram mudança líquida negativa e apenas três mantiveram a mesma área basal entre os períodos avaliados (Tabela 4).

Entre as 15 espécies que apresentaram redução em área basal, uma foi classificada como Pioneira, 12 foram classificadas como Clímax Exigentes em Luz e duas como Clímax Tolerante à Sombra. Dentre as 67 que apresentaram incremento em área basal, oito foram classificadas como Pioneiras, 50 foram classificadas como Clímax Exigentes em Luz e nove foram classificadas como Clímax Tolerante à Sombra. Dentre as três espécies que não apresentaram mudança líquida, todas foram classificadas como Clímax

TABELA 3: Tabela de contingência contendo o número de espécies observado e esperado (entre parênteses), de acordo com o teste de qui-quadrado $\left(\chi^{2}=4,3156, p=0,365\right)$, considerando as guildas de regeneração e as tendências de dinâmica, para populações de espécies arbóreas, em um fragmento de Floresta Ombrófila Mista, em Lages - SC.

TABLE 3: Contingence table containing the number observed and expected, between parentheses, of species, according to chi-square test $\left(\chi^{2}=4,3156, p=0,365\right)$, considering the regeneration guild and the dynamics trends, for tree species populations, in an Araucaria Forest fragment, in the municipality of Lages, SC state.

\begin{tabular}{cccc}
\hline Número de espécies & \multicolumn{4}{c}{ Guildas de Regeneração } & \\
\hline Tendência de dinâmica & P & CEL & CTS \\
\hline$\uparrow$ & $2(3,39)$ & $25(24,47)$ & $5(4,14)$ \\
$\leftrightarrow$ & $4(3,81)$ & $26(27,53)$ & $6(4,66)$ \\
$\downarrow$ & $3(1,80)$ & $14(13,00)$ & $0(2,20)$ \\
\hline
\end{tabular}

Em que: $\mathrm{P}=$ Pioneira; $\mathrm{CEL}=$ Clímax Exigente em Luz; $\mathrm{CTS}=$ Clímax Tolerante à Sombra. 
Exigentes em Luz.

Da mesma forma, de acordo com o teste de qui-quadrado $\left(\chi^{2}=1,3251, p=0,8571\right)$, também não foi observada associação significativa entre os padrões de dinâmica das espécies e a classificação em guildas de regeneração.

As proporções do número de indivíduos e de espécies nas guildas de regeneração não se alteraram significativamente entre os anos de avaliação (Tabela 5), sendo que a maioria dos indivíduos e espécies pertenceram ao grupo Clímax Exigentes em Luz, seguida, em menor proporção, por Clímax Tolerantes à Sombra e, finalmente, as Pioneiras.

Em florestas tropicais úmidas isentas de distúrbios severos, geralmente são registradas poucas mudanças na densidade e na composição de espécies, sugerindo uma aparente estabilidade (SWAINE et al., 1987; LAURANCE et al., 2006). Segundo Machado e Oliveira-Filho (2010), essa condição de "estabilidade" pode corresponder à fase clímax ou madura de uma floresta.

Em relação à representatividade das guildas de regeneração, o mesmo padrão encontrado no presente estudo, de maior abundância de espécies classificadas como CEL, foi observado por trabalhos realizados em fragmentos de Floresta Ombrófila Mista (ABREU, 2012; CHAGAS et al., 2001; PAULA et al., 2004).

TABELA 4: Tabela de contingência contendo o número de espécies em relação à dinâmica de área basal observado e esperado, entre parênteses, de acordo com o teste de qui-quadrado $\left(\chi^{2}=1,3251\right.$, $p=0,8571)$ considerando as guildas de regeneração e as tendências de dinâmica, para populações de espécies arbóreas, em um fragmento de Floresta Ombrófila Mista, em Lages SC.

TABLE 4: Contingence table containing the observed and expected, between parentheses, number of species, in relation to dynamics of basal area, according to chi-square test $\left(\chi^{2}=1,3251\right.$, $p=0,8571)$, considering the regeneration guild and the dynamics trends for tree species populations, in an Araucaria Forest fragment, in the municipality of Lages, SC state.

\begin{tabular}{cccc}
\hline Área Basal & \multicolumn{3}{c}{ Guildas de Regeneração } \\
\hline Tendência de dinâmica & Pioneira & CEL & CTS \\
\hline$\uparrow$ & $8(7,09)$ & $50(51,2)$ & $9(8,67)$ \\
$\leftrightarrow$ & $0(0,32)$ & $3(2,29)$ & $0(0,39)$ \\
$\downarrow$ & $1(1,59)$ & $12(11,5)$ & $2(1,94)$ \\
\hline
\end{tabular}

Em que: $\mathrm{P}=$ Pioneira; $\mathrm{CEL}=$ Clímax Exigente em Luz; $\mathrm{CTS}=$ Clímax Tolerante à Sombra.

TABELA 5: Tabela contendo a porcentagem de indivíduos e de espécies nos diferentes anos de avaliação em relação às guildas de regeneração, para populações de espécies arbóreas, em um fragmento de Floresta Ombrófila Mista, em Lages - SC.

TABLE 5: Table containing the percentage of individuals and species in different evaluated years in relation to regeneration guilds, for tree species populations, in an Araucaria Forest fragment, in the municipality of Lages, SC state.

\begin{tabular}{|c|c|c|c|c|c|c|c|c|}
\hline \multirow{2}{*}{ Guildas de regeneração } & \multicolumn{4}{|c|}{ Número de Indivíduos } & \multicolumn{4}{|c|}{ Número de Espécies } \\
\hline & 2008 & 2012 & $\chi^{2}$ & $p$ & 2008 & 2012 & $\chi^{2}$ & $p$ \\
\hline $\mathrm{P}$ & 7,14 & 6,88 & 0,0603 & 0,806 & 10,71 & 10,84 & 0 & 1 \\
\hline CEL & 79,13 & 78,18 & 0,4384 & 0,5079 & 76,19 & 75,90 & 0 & 1 \\
\hline CTS & 13,73 & 14,94 & 0,9998 & 0,3174 & 13,10 & 13,25 & 0 & 1 \\
\hline Total & 100 & 100 & & & 100 & 100 & & \\
\hline
\end{tabular}

Em que: $\mathrm{P}=$ Pioneira; $\mathrm{CEL}=$ Clímax Exigente em Luz; $\mathrm{CTS}=$ Clímax Tolerante à Sombra; $\chi 2=$ Qui-quadrado; $p=$ valor de $\mathrm{p}$ de acordo com o teste de proporção. 
Paula et al. (2004) comentam que a baixa representatividade florística das espécies pioneiras e o maior número de secundárias tardias, que, junto com as secundárias iniciais, são análogas às Clímax Exigente em Luz, exprimem um estádio intermediário de desenvolvimento sucessional da floresta. Porém, se for considerada a área basal observada em $2008\left(36,16 \mathrm{~m}^{2} \cdot \mathrm{ha}^{-1}\right)$ e $2012\left(39,17 \mathrm{~m}^{2} \cdot \mathrm{ha}^{-1}\right)$, o fragmento estudado pode ser considerado como em avançado estágio de regeneração, de acordo com a Resolução CONAMA 04/1994.

\section{CONCLUSÕES}

A análise dos resultados indica, por meio do incremento da densidade do número de indivíduos e área basal, que a floresta se encontra em fase de instabilidade estrutural, em um processo de "construção silvigenética". Já os dados referentes à florística e guildas de regeneração sugerem que a floresta está próxima de seu clímax florístico sucessional. Esta inferência se dá a partir da constatação da: i) estabilidade do número de espécies, indicada pela baixa substituição florística entre os quatro anos avaliados, representada pela redução de apenas uma espécie; ii) ausência de associação significativa entre os padrões de dinâmica das populações e as guildas de regeneração; iii) manutenção da participação relativa, em número de indivíduos e de espécies, das diferentes guildas entre os anos, e iv) dominância das espécies Clímax Exigentes em Luz.

Ressalta-se a necessidade de estudos que considerem uma maior escala de tempo, para verificar possíveis flutuações de parâmetros populacionais e a influência de variáveis ambientais sobre a dinâmica florestal.

\section{AGRADECIMENTOS}

À CAPES, pela concessão de bolsa de mestrado ao primeiro autor desse trabalho, e ao CNPq, pela concessão de bolsa de produtividade em pesquisa ao segundo e ao terceiro autor.

\section{REFERÊNCIAS}

ABREU, T. A. L. Dinâmica da vegetação arbustivo-arbórea no período de 14 anos em uma floresta de vale, Mato Grosso, Brasil. 2012. 88 f. Dissertação (Mestrado em Ciências Florestais) - Universidade de Brasília, Brasília, 2012.

ANGIOSPERM PHYLOGENY GROUP III. An update of the Angiosperm Phylogeny Group classification for the orders and families of flowering plants: APG III. Botanical Journal of the Linnean Society, Hoboken, v. 161, n. 2, p. 105-121, 2009.

CHAGAS, R. K. et al. Dinâmica de populações arbóreas em um fragmento de Floresta Estacional Semidecidual Montana em Lavras, Minas Gerais. Revista Árvore, Viçosa, MG, v. 25, n. 1, p. 39-57, 2001. DURIGAN, M. E. Florística, dinâmica e análise protéica de uma Floresta Ombrófila Mista em São João do Triunfo-PR. 1999. 125 f. Dissertação (Mestrado em Engenharia Florestal) - Universidade Federal do Paraná, Curitiba, 1999.

FIGUEIREDO-FILHO, A. et al. Crescimento, mortalidade, ingresso e distribuição diamétrica em floresta ombrófila mista. Floresta, Curitiba, v. 40, n. 4, p. 763-776, 2010.

FORMENTO, S.; SCHORN, L. A.; RAMOS, R. A. Dinâmica estrutural arbórea de uma Floresta Ombrófila Mista em Campo Belo do Sul. Cerne, Lavras, v. 10, n. 2, p. 196-212, 2004.

HALLÉ, F.; OLDEMAN, R. A. A.; TOMLINSON, P. B. Tropical trees and forests. Berlin: SpringerVerlag, 1978. $483 \mathrm{p}$.

HIGUCHI, P. et al. Influência de variáveis ambientais sobre o padrão estrutural e florístico do componente arbóreo, em um fragmento de Floresta Ombrófila Mista Montana em Lages, SC. Ciência Florestal, Santa Maria, v. 22, n. 1, p. 7-90, 2012.

IBGE. Manual técnico da vegetação brasileira. 2. ed. Rio de Janeiro: IBGE, 2012. (Série Manuais técnicos em geociências, 1).

KORNING, J.; BALSLEV, H. Growth and mortality of trees in Amazonian tropical rain forest in Ecuador. Journal of Vegetation Science, Knivsta, v. 4, n. 1, p. 77-86, 1994. 
LAURANCE, F. W. et al. Rapid decay of tree-community composition in Amazonian forest fragments. PNAS, Washington, v. 103, n. 50, p. 19010- 19014, 2006.

LEYSER, G. et al. Regeneração de espécies arbóreas e relações com o componente adulto em uma floresta estacional no vale do rio Uruguai, Brasil. Acta Botanica Brasilica, Feira de Santana, v. 26, n. 1, p. 74-83, 2012.

LIEBERMAN, D. et al. Mortality patterns and stand turnover rates in a wet tropical forest in Costa Rica. Journal of Ecology, Hoboken, v. 73, n. 3, p. 915-924, 1985.

LINDENMAIER, D. S.; BUDKE, J. C. Florística, diversidade e distribuição espacial das espécies arbóreas em uma floresta estacional na bacia do rio Jacuí, Sul do Brasil. Pesquisas, Botânica, São Leopoldo, v. 57, p. 193-216, 2006.

LINGNER, D. V. et al. Caracterização da estrutura e da dinâmica de um remanescente de Floresta de Araucária no Planalto Catarinense. Pesquisa Florestal Brasileira, Colombo, n. 55, p. 55-66, 2007.

MACHADO, E. L. M. Heterogeneidade espacial e temporal em um fragmento de floresta estacional em Lavras, MG. 2005. 101 f. Dissertação (Mestrado em Engenharia Florestal) - Universidade Federal de Lavras, Lavras, 2005.

MACHADO, E. L. M.; OLIVEIRA-FILHO, A. T. Spatial patterns of tree community dynamics are detectable in a small (4 ha) and disturbed fragment of the Brazilian Atlantic forest. Acta Botanica Brasilica, Feira de Santana, v. 24, n.1, p. 250-261, 2010.

MOGNON, F. Dinâmica do estoque de carbono como serviço ambiental prestado por um fragmento de Floresta Ombrófila Mista Montana localizada no sul do estado do Paraná. 2011. 142 f. Dissertação (Mestrado em Engenharia Florestal) - Universidade Federal do Paraná, Curitiba, 2011.

MOSCOVICH, F. A. Dinâmica de crescimento de uma Floresta Ombrófila Mista em Nova Prata, RS. 2006. 130 f. Tese (Doutorado em Engenharia Florestal) - Universidade Federal de Santa Maria, Santa Maria, 2006.

PAULA, A. et al. Sucessão ecológica da vegetação arbórea em uma Floresta Estacional Semidecidual, Viçosa/MG. Acta Botanica Brasilica, Feira de Santana, v. 18, n. 3, p. 407-424, 2004.

PRIMACK, R. B. et al. Growth rates and population structure of Moraceae trees in Sarawak, East Malaysia. Ecology, Washington, v. 66, n. 2, p. 577-588, 1985.

R DEVELOPMENT CORE TEAM. R: A language and environment for statistical computing. R Foundation for Statistical Computing, Vienna, Austria, 2012. Disponível em $<$ http://www.R-project.org $>$ Acesso em: 14 mar. 2013.

ROIK, M. et al. Incremento diamétrico e em área basal de espécies arbóreas em fragmento de Floresta Ombrófila Mista. In: CONGRESSO FLORESTAL PARANAENSE, 4., 2012, Curitiba. Anais... Curitiba, 2012.

SANQUETTA, C. R. Métodos de determinação de biomassa florestal. In: SANQUETTA, C. R. et al. (Orgs.). As florestas e o carbono. Curitiba: UFPR, 2002. p. 119-140.

SANQUETTA, C. R.; DALLA CORTE, A. P.; EISFELD, R. L. Crescimento, mortalidade e recrutamento em duas florestas de araucária (Araucaria angustifolia (Bert.) O. Ktze.). no Estado do Paraná, Brasil. Revista Ciências Exatas e Naturais, Guarapuava, v. 5, n. 1, p. 101-112, 2003.

SERRÃO, D. R.; JARDIM, F. C. S; NEMER, T. C. Sobrevivência de seis espécies florestais em uma área explorada seletivamente no município de Moju, Pará. Cerne, Lavras, v. 9, n. 2, p. 153-163, 2003.

SHEIL, D.; MAY, R. M. Mortality and recruitment rate evaluations in heterogeneous tropical forests. Journal of Ecology, Hoboken, v. 84, n. 1, p. 91-100, 1996.

SWAINE, M. D.; LIEBERMAN, D.; PUTZ, F. E. The dynamics of tree populations in tropical forest: a review. Journal of Tropical Ecology, Cambridge, v. 3, p. 359-366, 1987. 